\title{
Serum eotaxin-1 is increased in extremely-low-birth-weight infants with bronchopulmonary dysplasia or death
}

\author{
Jegen Kandasamy ${ }^{1}$, Claire Roane ${ }^{1}$, Alexander Szalai ${ }^{2}$ and Namasivayam Ambalavanan
}

BACKGROUND: Early systemic inflammation in extremelylow-birth-weight (ELBW) infants is associated with an increased risk of bronchopulmonary dysplasia (BPD). Our objective was to identify circulating biomarkers and develop prediction models for BPD/death soon after birth.

METHODS: Blood samples from postnatal day 1 were analyzed for C-reactive protein (CRP) by enzyme-linked immunosorbent assay and for 39 cytokines/chemokines by a multiplex assay in 152 ELBW infants. The primary outcome was physiologic BPD or death by 36 wk. CRP, cytokines, and clinical variables available at $\leq 24 \mathrm{~h}$ were used for forward stepwise regression and Classification and Regression Tree (CART) analysis to identify predictors of BPD/death.

RESULTS: Overall, 24\% developed BPD and 35\% died or developed BPD. Regression analysis identified birth weight and eotaxin (CCL11) as the two most significant variables. CART identified $\mathrm{FiO}_{2}$ at $24 \mathrm{~h}$ (11\% BPD/death if $\mathrm{FiO}_{2} \leq 28 \%, 49 \%$ if $>28 \%$ ) and eotaxin in infants with $\mathrm{FiO}_{2}>28 \%$ (29\% BPD/death if eotaxin was $\leq 84 \mathrm{pg} / \mathrm{ml} ; 65 \%$ if $>84$ ) as variables most associated with outcome.

CONCLUSION: Eotaxin measured on the day of birth is useful for identifying ELBW infants at risk of BPD/death. Further investigation is required to determine if eotaxin is involved in lung injury and pathogenesis of BPD.

B ronchopulmonary dysplasia (BPD) is a chronic lung disease of infancy secondary to disordered postnatal bronchoalveolar and pulmonary vascular development and maturation. A third of extremely-low-birth-weight (ELBW) infants develop BPD, making it one of the most common morbidities following preterm birth (1). BPD has a multifactorial etiology associated with many postnatal risk factors including prolonged mechanical ventilation, exposure to hyperoxia, infections, and differences in genetic predisposition (2). The risk of BPD is also increased by prenatal factors including maternal chorioamnionitis, preeclampsia and intrauterine growth retardation (3). Excessive inflammation or a predisposition to accentuation of inflammation for a given stimulus is a common theme for many of these predisposing factors.

Cytokines, a large group of immune-modulatory proteins that also perform nonimmune functions, are major mediators of inflammatory pathways. A number of these cytokines including TNF- $\alpha$, IL- $1 \beta$, and IL- 6 are increased in tracheal aspirates of infants with BPD, and increased tracheal IL- 6 and IL-8 concentrations have been shown to precede the development of BPD $(4,5)$. Proinflammatory cytokines such as IL-1, IL-6, and IL-17 increase the production of C-reactive protein (CRP), an acute phase reactant that decreases surfactant activity in a dose-dependent manner (6). We have shown recently that CRP is a biomarker for BPD/death in ELBW infants (7).

Our hypothesis in this study was that specific serum cytokine levels available by $24 \mathrm{~h}$ of life would help in early identification of ELBW infants at risk for BPD or death. We also attempted to develop predictive models for BPD risk stratification by adding cytokine data to clinical variables available in the first postnatal day. We used a combined outcome of death or BPD in order in order to include infants who died soon after birth before they could be defined as having BPD at $36 \mathrm{wk}$ age (i.e., death is a competing outcome for BPD; most deaths in extremely preterm infants are due to respiratory causes, even if the processes leading to death and BPD are sometimes different, e.g., sepsis (8)). Our choice of including death together with BPD to form a combined primary outcome identifies a group of ELBW infants at high risk for poor outcome.

\section{RESULTS}

\section{Clinical Characteristics}

Of 267 eligible infants, informed consent was obtained from mothers/caregivers of 152 infants, and these 152 infants were then enrolled in the study and followed to $36 \mathrm{wk}$ postmenstrual age. $47 \%$ of the infants enrolled were white, and $53 \%$ were black. $42 \%$ were male (Table 1 ). The mean gestational age (GA) of infants enrolled was $25.2 \pm 2.8 \mathrm{wk}$; mean birth weight (BW) was $720.16 \pm 147 \mathrm{~g} .61 \%$ of all infants enrolled were delivered by cesarean section. $91 \%$ of all mothers received at least one dose of antenatal steroids. Thirty-five (23\%) infants were small for GA (SGA), and 117 (77\%) were appropriate for GA (AGA). $52 \%$ of our infant cohort was mechanically ventilated at $24 \mathrm{~h}$ of age, another $14 \%$ required continuous positive airway pressure, $14 \%$ required supplemental oxygen only, and the rest were stable in room air. Overall, 35 infants (23\%) developed BPD, 18 infants (11\%) died, and 99 (66\%) survived to 36 wk without BPD (Table 1 ). 
Table 1. Clinical characteristics of enrolled infants

\begin{tabular}{|c|c|c|c|c|}
\hline Variable & Overall & Survival without BPD & BPD and/or death & $P^{a}$ \\
\hline$n(\%)$ & $152(100)$ & $99(65)$ & $53(35)$ & \\
\hline Birth weight, mean $\pm S D, g$ & $720 \pm 147$ & $753 \pm 142$ & $658 \pm 136$ & $<0.001$ \\
\hline Small for gestational age, $n$ (\%) & $35(23)$ & $19(19)$ & $16(30)$ & 0.12 \\
\hline White ethnicity, $n$ (\%) & $71(47)$ & $48(48)$ & $23(43)$ & 0.69 \\
\hline Complete course of prenatal steroids, $n(\%)$ & $82(54)$ & $62(63)$ & $20(38)$ & 0.01 \\
\hline Clinical chorioamnionitis, $n$ (\%) & $15(10)$ & $12(12)$ & $3(6)$ & 0.32 \\
\hline Histological chorioamnionitis, $n$ (\%) & $53(35)$ & $36(37)$ & $17(32)$ & 0.64 \\
\hline Use of maternal antibiotics, $n(\%)$ & $113(74)$ & $80(81)$ & $33(62)$ & 0.02 \\
\hline 5-min Apgar scores, median (IQR) & $7(5,7)$ & $7(6,7)$ & $6(4,7)$ & 0.01 \\
\hline Mechanical ventilation at 36 wk PMA, $n$ (\%) & $9(6)$ & $2(2)$ & $7(13)$ & 0.01 \\
\hline Mechanical ventilation duration by $36 \mathrm{wk} P M A$, mean $\pm S D$, days & $10.2 \pm 17.4$ & $7.1 \pm 11.9$ & $16.3 \pm 23.8$ & 0.002 \\
\hline Oxygen supplementation duration by $36 \mathrm{wk} P M A$, mean $\pm \mathrm{SD}$, days & $30.6 \pm 30.7$ & $26.2 \pm 28.0$ & $39.1 \pm 34.0$ & 0.01 \\
\hline $\mathrm{FiO}_{2}$ at 36 wk PMA, median (IQR) & $21(21)$ & $21(21)$ & $25(21,39)$ & 0.003 \\
\hline Highest $\mathrm{FiO}_{2}$ at 36 wk PMA, median (IQR) & $21(21,25)$ & $21(21)$ & $27(21,40)$ & 0.001 \\
\hline Absolute eosinophil count, median (IQR), cells/ $\mu \mathrm{l}$ & $140(43,251)$ & $132(32,251)$ & $18(79,530)$ & 0.03 \\
\hline
\end{tabular}

The median postnatal age of the group of infants who died was $11 \mathrm{~d}$ (IQR: 5-20). Eleven of these infants (61\%) were black. They were also more immature compared to infants who survived (24 vs. $25 \mathrm{wk}, 620$ vs. $710 \mathrm{~g}$, both $P<0.001)$. Eight of these infants died due to respiratory distress syndrome, three due to sepsis, and another two due to respiratory distress syndrome with sepsis. Four deaths were due to gastrointestinal causes such as necrotizing enterocolitis and spontaneous intestinal perforation. One infant died at a postnatal age of $77 \mathrm{~d}$ due to unclassified causes.

\section{Cytokines}

Of the 39 cytokines (Table 2) that were measured, only eotaxin-1 (CCL11) was higher in infants who developed BPD compared to infants who survived without BPD (median (2575th centile) $\mathrm{pg} / \mathrm{ml}$ : $112(40-247)$ vs. 48 (16-108), $P=0.02)$. Infants who died also had higher serum eotaxin concentration when compared to infants who survived without BPD (median (25-75th centile) pg/ml: 103 (25-152) vs. 48 (16-108), $P=$ 0.03 ). These differences in eotaxin concentration persisted when infants who developed BPD or died before $36 \mathrm{wk}$ postmenstrual age (PMA) were compared to infants who survived without BPD (median (25-75th centile) pg/ml: 108 (31-198) vs. 48 (16-108), $P=0.017$ ) (Figure 1). MDC (macrophagederived cytokine; CCL22) was also higher in infants who developed BPD or died (median (25-75th centile) pg/ml: 826 (493-1162) vs. $627(30,1,001), P=0.035)$. Neither eotaxin nor MDC correlated strongly with BW or GA $(r<0.2, P>0.05)$.
Eotaxin correlated strongly with GM-CSF, IL-6, MCP-1 $(r>$ $0.7, P<0.001)$ and moderately with IL- $1,-2,-3,-7,-8,-9,-10$, IFN- $\gamma$, Fractalkine, MCP-3, MIP- 1 , and TNF- $\alpha(r=0.4-0.7, P$ $<0.001)$; in contrast, MDC was only weakly correlated with other cytokines ( $r$ values of $0.28,0.002,0.066$ with GM-CSF, IL-6, and MCP-1 respectively, all $P$ values $>0.5$ ).

Regression analysis was carried out using clinical variables considered relevant to BPD pathogenesis that are usually available by $24 \mathrm{~h}$ of life (BW, GA, ethnicity, sex, multiple birth, chorioamnionitis, maternal antenatal steroid use, Apgar scores at 1 and $5 \mathrm{~min}, \mathrm{CRP}$, and the 39 cytokines; BPD and/or death was the dependent variable. Birth weight and eotaxin-1 (partial $R^{2}$ of 0.13 and 0.09 , respectively) were identified as the two most significant variables in this analysis.

Stepwise forward classification and regression analysis (CART) using BPD and/or death as outcome variable identified $\mathrm{FiO}_{2}$ at $24 \mathrm{~h}\left(11 \% \mathrm{BPD} /\right.$ death if $\mathrm{FiO}_{2}<28 \%, 49 \%$ if $>28 \%$ ) as the only significant factor that was predictive of risk for BPD and/or death (Figure 2). Of the cytokines that were analyzed, only eotaxin-1 was identified as a significant predictor for risk of $\mathrm{BPD} /$ death in infants with $\mathrm{FiO}_{2}$ $>28 \%(29 \% \mathrm{BPD} /$ death if eotaxin- 1 was $<84 \mathrm{pg} / \mathrm{ml} ; 65 \%$ if $>84)$. The thresholds for $\mathrm{FiO}_{2}$ at $24 \mathrm{~h}\left(\mathrm{FiO}_{2}<28 \%\right.$ vs. $\left.>28 \%\right)$ and eotaxin- $1(<84 \mathrm{pg} / \mathrm{ml}$ vs. $>84 \mathrm{pg} / \mathrm{ml})$ were identified by the CART analysis. No other cytokine, including MDC, improved predictive ability of this model for $\mathrm{BPD} /$ death in ELBW infants. 
Articles | Kandasamyet al.

Table 2. CRP and serum cytokine concentrations measured on postnatal day 1, stratified according to outcome status

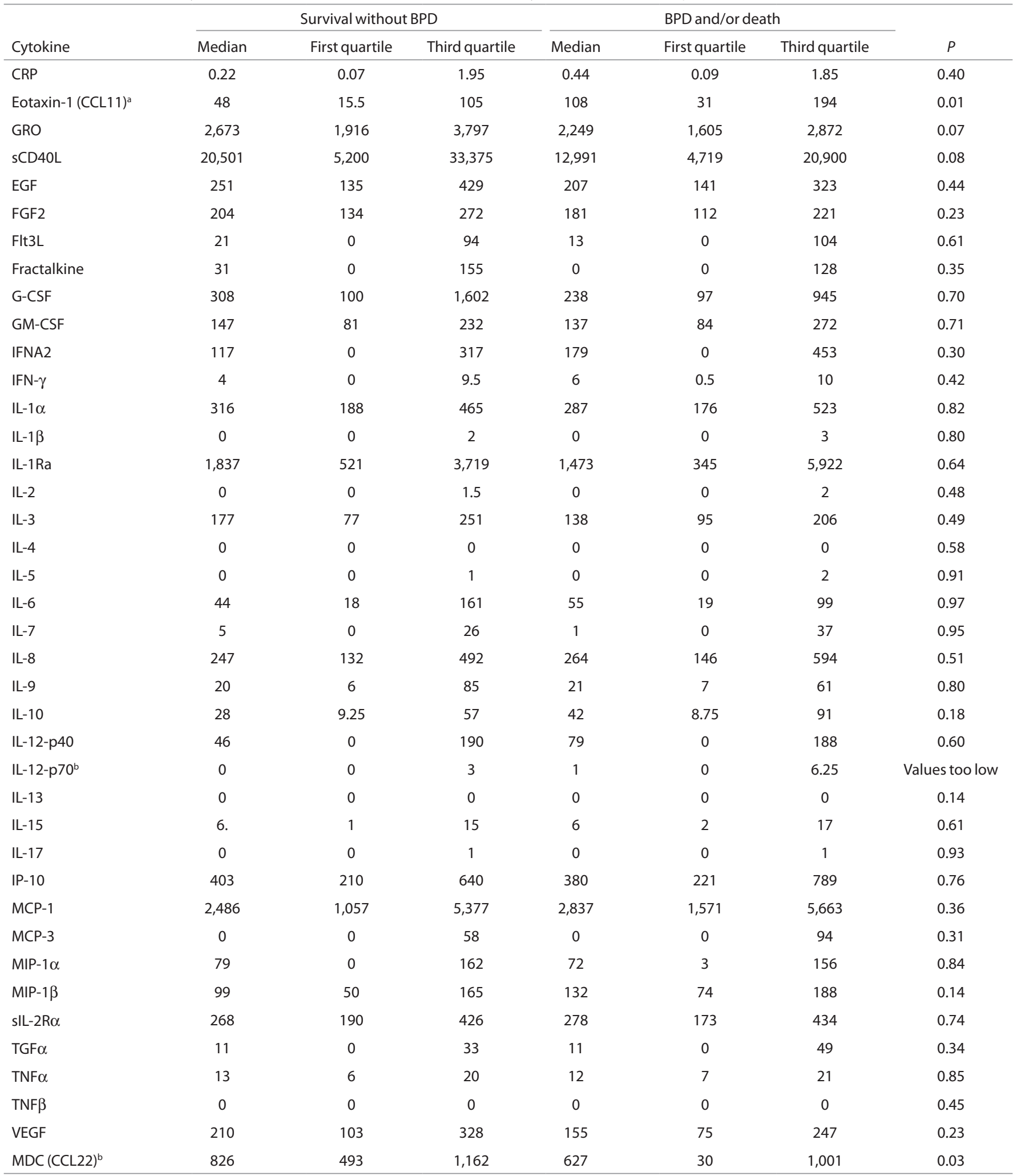

All cytokine levels are in $\mathrm{pg} / \mathrm{ml}$. All $P$ values were calculated using the Mann-Whitney $\mathrm{U}$-test.

aStatistically significant. ${ }^{b} P$ values were close to threshold of measurement

BPD, bronchopulmonary dysplasia; CRP, C-reactive protein; CXCL1, CXC ligand 1; FGF2, fibroblast growth factor 2; Flt-3L, Fms-like tyrosine kinase 3 ligand; G-CSF, granulocyte-colony stimulating factor; GM-CSF, granulocyte macrophage-colony stimulating factor; MCP-1, monocyte chemoattractant protein-1; MDC, macrophage-derived chemokine; MIP-1 $\alpha$,

macrophage inflammatory protein-1 $\alpha$; sCD40L, surface expressed; sIL-2R $\alpha$, soluble IL-2R $\alpha$; VEGF, vascular endothelial growth factor. 
Subgroup analyses of cytokines based on chorioamnionitis, pregnancy-induced hypertension and intrauterine growth retardation status were also carried out. There were no significant differences in cytokine levels between SGA and AGA infants. Our study had 35 SGA infants of whom 16 (45\%) developed BPD or died, compared to 38 of 117 infants in the AGA group (32\%). Eotaxin-1 and MDC were higher in AGA infants who died or developed BPD compared to those who survived (median (25-75th centile) pg/ml: eotaxin: 118.00 (54-248.00) vs. 49 (17-104), $P=0.003$, MDC: $1059(629-1,438)$ vs. 580 (277-960), $P=0.001)$. Among SGA infants, there were no significant differences in cytokine levels between the two groups. Infants born to mothers with pregnancy-induced hypertension had similar eotaxin levels compared to infants born to mothers without PIH (median (25-75th centile) pg/ml: 51 (12-124) vs. 67 (20-129), $P=0.71)$. Infants born to mothers who had chorioamnionitis had similar levels of all cytokines that were

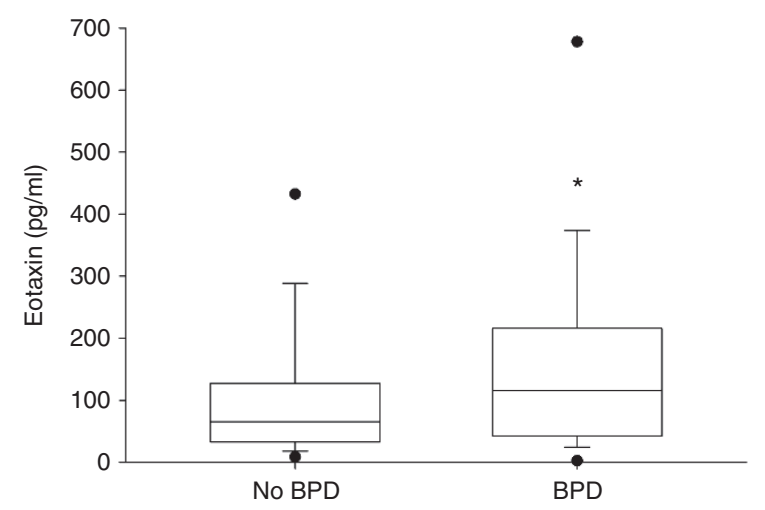

Figure 1. Serum eotaxin concentrations on postnatal day 1, comparing ELBW infants who survived without bronchopulmonary dysplasia (BPD) to infants who died or develop BPD. Box and whiskers plot indicates median, 25th-75th centiles (lower and upper borders of box), 5th-95th centiles (lower and upper whiskers) and outliers (black dots). ${ }^{*} P<0.05$ vs. "No BPD" group. measured compared to infants whose mothers did not (median (25-75th centile) pg/ml: $46(0-125)$ vs. $55(25-124), P=0.45)$.

\section{Eosinophil Counts and Outcomes}

As the primary role of eotaxin (the only cytokine identified as significant in the CART analysis) is eosinophil chemotaxis and activation, a post-hoc analysis of absolute eosinophil counts was carried out. Absolute eosinophil count (Figure 3) was significantly higher in infants who died or developed BPD (median (25-75th centile) cells $/ \mathrm{mm}^{3}: 189$ (79-530) vs. 140 (43-251), $P<0.05)$. However, there was no correlation between eotaxin-1 concentrations and absolute eosinophil counts $(r=0.03, P>0.5)$.

\section{Clinical Characteristics and Outcomes}

In addition to cytokine measurements, certain clinical variables were also correlated with BPD and/or death. $63 \%$ of mothers of infants who survived without BPD received a complete course of prenatal steroids compared to 33\% of mothers whose infants died and $38 \%$ of mothers whose infants developed BPD $(P<0.05)$. Infants who developed BPD weighed less at birth compared to those that survived without BPD $(P<$ $0.001)$. However there was no significant difference in mean GA between the two groups, suggesting that growth restriction (lower BW for similar GA) was a contributor to BPD (Table 1).

At $24 \mathrm{~h}$ of age, median supplemental oxygen requirements were 45,36 , and $30 \%$ for infants who died, survived with BPD, and survived without BPD respectively $(P<0.001)$. More infants who died also required invasive mechanical ventilation compared to those who survived with, or without $\operatorname{BPD}(89,69$, and $39 \%$ respectively, $P<0.001$ or those who did not have BPD. Incidence of pulmonary hemorrhage was higher for infants who died (33\%) compared to infants who survived with BPD $(11 \%)$ or infants who survived without BPD $(7 \%)(P<0.05)$. As would be expected, the cumulative duration of supplemental oxygen therapy was significantly higher at $36 \mathrm{wk}$ PMA for

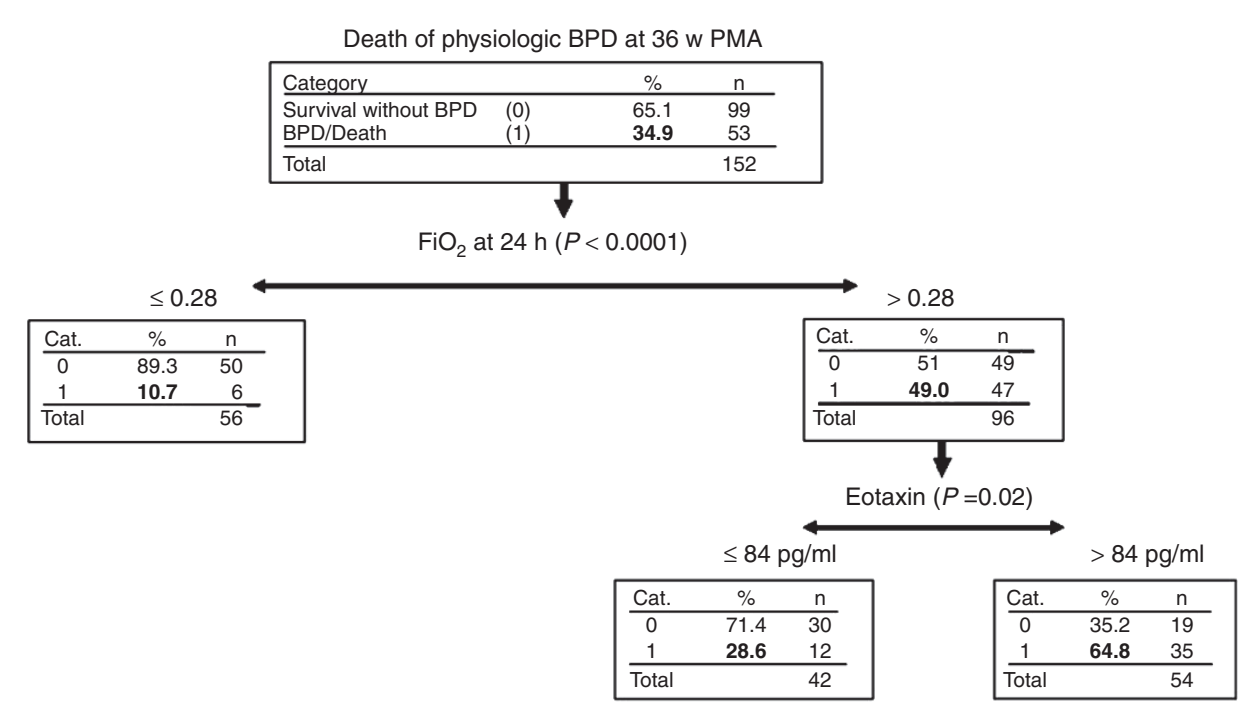

Figure 2. Classification and regression tree model for bronchopulmonary dysplasia (BPD)/death by 36 wk postmenstrual age. The percentage of BPD/ death at each node is indicated in bold font. 


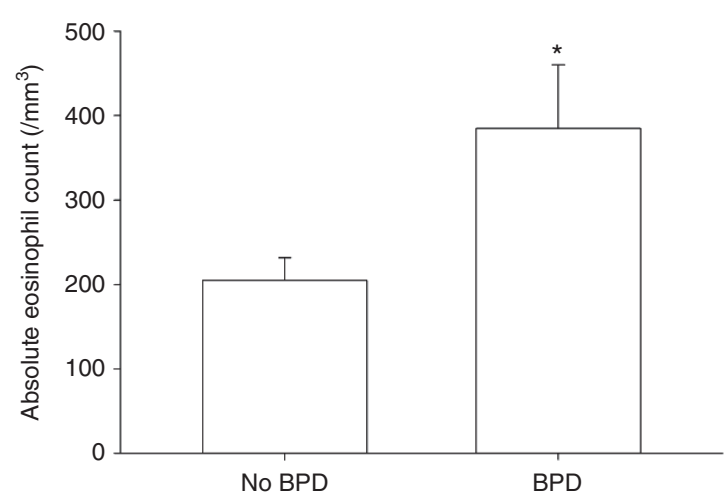

Figure 3. Absolute eosinophil counts on postnatal day 1 in infants who survived without bronchopulmonary dysplasia (BPD), compared to those who died or developed BPD (mean \pm SEM; ${ }^{*} P<0.05$ vs. "No BPD" group).

infants who developed BPD or died compared to infants who did not have BPD (Table 1). They were also mechanically ventilated for longer duration by $36 \mathrm{wk}$ PMA $(16.33 \pm 24 \mathrm{~d}) \mathrm{com}-$ pared to BPD-free survivors $(7.1 \pm 12 \mathrm{~d}$ ). ELBW infants who died or developed BPD in our study had a cumulative duration of need for supplemental oxygen of $39 \pm 34 \mathrm{~d}(6 \mathrm{wk})$, a duration shorter than their mean duration to $36 \mathrm{wk}$ PMA (11wk). We believe this was due to the quick resolution of initial respiratory distress syndrome, followed by a more insidious pattern of waxing and waning oxygen requirements in later life-a pattern reflective of the "new" BPD phenotype that is more often seen these days, due to increased exposure to antenatal steroids and surfactant. It may also be reflective of the fact that infants who died in our study had a mean age of $16 \mathrm{~d}$, which means they received supplemental oxygen for a shorter duration than the subgroup of survivors with BPD who received oxygen for a longer duration $(59 \pm 22 \mathrm{~d})$.

There was no significant association between maternal clinical or histologic chorioamnionitis and the risk for BPD or death, although 9 of 18 infants who died (50\%) were exposed to histological chorioamnionitis (50\%), compared to 25 (22\%) infants who developed BPD, and $37(37 \%)$ of infants who survived without BPD $(P=0.12)$. Early onset sepsis, often associated with maternal chorioamnionitis, was also similar in incidence between infants who died, developed BPD and those who survived without BPD by $36 \mathrm{wk}$ PMA. The incidence of blood culture positive late-onset sepsis, on the other hand, was significantly higher for infants who developed BPD or died compared to infants who survived without BPD (57 vs. $33 \%, P=0.003$ ). Interestingly, the incidence of late onset sepsis was lower for infants who died than for infants who developed BPD (39 vs. $66 \%$ ), most likely due to their early death. No differences were noted in the incidence of meningitis between infants who died, or survived, with or without BPD.

\section{DISCUSSION}

The aims of the current study were to identify specific cytokines and/or cytokine profile patterns in ELBW infants soon after birth that would be useful to predict their risk for BPD and/or death. Our CART model using clinical factors available on the first postnatal day showed that only $\mathrm{FiO}_{2}$ was useful as a predictor of this risk. Of the 40 mediators (CRP and 39 cytokines) that were measured, only serum eotaxin-1 (CCL11) measured on the day of birth added to the predictive ability of this model. Other cytokines such as IL- $1 \beta$ and TNF- $\alpha$ as well as CRP were similarly distributed among infants who either died or developed BPD and those that survived without BPD. Eotaxin-1 levels correlated strongly with some of these cytokines including GM-CSF, IL-6, and MCP-1, but poorly with BW or GA. We also found that while eosinophil counts in the first few days of life were significantly higher in infants at risk of death or BPD, there was no correlation between eotaxin-1 levels and absolute eosinophil count.

Cytokines have been previously studied with regard to BPD and/or death in large cohorts of ELBW infants. A recent study conducted by the Neonatal Research Network (NRN) evaluated serum profiles of 25 different cytokines between the 3rd and 28th days of life in a cohort of 1067 ELBW infants and showed that higher concentrations of cytokines such as IL-1 $\beta$, IL-6, IL-8, and IL-10, and IFN- $\gamma$ preceded BPD/death; as did lower concentrations of other cytokines (IL-17, regulated on activation, normal T-cell expressed and secreted (RANTES), and TNF- $\beta$ ) (9). The extremely low GA neonates (ELGAN) study analyzed 25 proteins including cytokines and chemokines at several time points in the first $2 \mathrm{wk}$ of life in a cohort of 932 infants (10). While no differences in cytokines were noted in the first week of life between infants with severe BPD and those with no BPD, increased inflammatory signaling was strongly evident in infants with severe BPD who had significantly higher levels of several cytokines by $14 \mathrm{~d}$ of life, including TNF- $\alpha$, IL-6, IL-8, and MCP-1 (10). In addition to TNF- $\alpha$ elevation, lower levels of RANTES were also seen more commonly in infants who developed BPD in both these large studies. Other outcomes related to ELBW infants such as cerebral palsy and retinopathy of prematurity have also been related to specific cytokine patterns (11-13).

Similar to these large cohorts, an important limitation of the current study is its observational nature which precludes derivation of cause-effect conclusions; hypothesis generation for future studies would be the key benefit. Another limitation is that only serum cytokines were measured. Many cytokines are produced in an autocrine manner in organs affected by inflammation, so measurement of tracheal aspirate or bronchoalveolar lavage fluid in addition to serum cytokine levels might have been more suitable to study the impact of cytokine associated inflammation on BPD. BPD, however, is primarily an alveolarinterstitial disease and tracheal aspirate or bronchoalveolar lavage is more representative of airway inflammation, making such an extrapolation also similarly imperfect.

Strengths of this study include the prospective nature of clinical and cytokine data collection, recruitment of a relatively large number of infants for a study of this nature and the addition of death prior to $36 \mathrm{wk}$ to BPD for the primary study outcome which removes confounding due to competing outcomes. The Luminex assay used in this study has low intraassay $(2-13 \%)$ and interassay (5-17\%) variability for cytokine 
and chemokine quantitation and has been well-validated (14). Thirty-nine cytokines were analyzed using this method, while previous studies based on other technologies measured fewer individual cytokines. Interestingly, this is the first study to our knowledge, to have measured eotaxin-1 levels (and MDC as well) in ELBW infants. Therefore elevations of these cytokines may have been present in the previously studied cohorts as well, but missed due to nonmeasurement. The CART method used to generate predictive models for death and/or BPD using cytokines and clinical factors is a rapid, simple and efficient method that is very useful in situations when there is insufficient previous knowledge about relationships between variables under study, a situation that is applicable to our existing knowledge about BPD and its inter-relationship with cytokines.

Eosinophils are best known for their roles in allergy and atopy-related diseases and antiparasitic defenses, but reports have shown positive correlation between peripheral blood eosinophilia, tissue eosinophilia in airways and alveoli, and eosinophil cationic protein (ECP; a marker for eosinophil activation) with increased risk for BPD (15-19). Such evidence provides a significant context for the primary biologic role of eotaxin - a chemokine and a prominent member of the Th2 cytokine family-a group that is known to be important in the pathogenesis of BPD, especially in the early postnatal period (9). Th2 cytokines that are found in increased concentrations in infants with BPD include IL-13 and TNF- $\alpha$, both known inducers of eotaxin production through the nuclear factor- $\kappa$ binding (NF-kB) pathway (9). IL-8 and MIP-1, two other cytokines shown to be elevated in bronchoalveolar lavage fluid of infants with BPD (20), are also primarily chemotactic in function. Thus it is possible that Th2 cytokine responses to inflammation could induce the chemotaxis of leukocytes to the lungs and their subsequent activation leading to lung injury and BPD. Eotaxin, as the primary recruiter of eosinophils to this inflammatory process, may play a key role in modulating this lung injury.

Eotaxin increases production of profibrotic cytokines such as TGF- $\beta$, MCP- 1 , and IL- 4 by eosinophils, mast cells and neutrophils in bleomycin-induced lung injury (21). Neutrophil influx may regulate inflammatory processes in the lung via eotaxin and other chemokines (22). Eotaxin also induces ROS production and increases oxidant damage in human endothelial cells (23). Thus, eotaxin may play roles in fibrogenesis and oxidant-induced lung damage, pathogenic processes known to occur to BPD. Eotaxin-1 and its major eotaxin receptor, the CCR3 ligand, are found in abundance in the developing lung where their expression causes increased proliferation and activation of extracellular-signal-regulated kinases (ERK1/2) in mid-trimester lung models (24). Thus, eotaxin may also modulate fetal lung development.

Eotaxin-1 measured on the day of birth is useful for identifying ELBW infants at risk of BPD/death. It is a potent eosinophil chemoattractant and activator, and eosinophilia and eosinophil activation are known to be correlated with severity of BPD. Although eotaxin-1 is not essential for lung development, it regulates airway epithelial proliferation in the developing lung and acute inflammatory lung injury. Additionally, genetic polymorphisms in the eotaxin gene, similar to those known to be involved in determining susceptibility to asthma in multiple populations (25), may also help explain upregulation of eotaxin expression in subgroups of infants and serve as markers of risk for BPD. Further investigation is required to determine if eotaxin- 1 is involved in BPD pathogenesis.

\section{METHODS}

\section{Study Design and Population}

This was a prospective cohort study conducted between 2005 and 2008 at the Regional Newborn Intensive Care Unit of the University of Alabama at Birmingham (UAB), Birmingham, AL. Approval was obtained from the institutional review board at UAB. Infants with BWs of $<1,000 \mathrm{~g}$ who survived beyond the first hour of life were eligible for enrollment in the study if informed parental consent could be obtained within the first $24 \mathrm{~h}$ after birth. Therefore, if mothers/ caregivers were not available or could not be approached (e.g., mother heavily sedated or very ill) or could not understand the informed consent document (non-English speaking), infants were considered not eligible. We excluded ELBW infants who were known to have congenital anomalies or syndromes.

\section{Clinical Data Collection}

Enrolled infants were followed through their hospital stay until the time of discharge or death. Prenatal, perinatal, and postnatal clinical variables were recorded by trained research staff. Clinical variables included BW (g), GA (wk), race, sex, clinical and histologic chorioamnionitis, use of antenatal steroids, Apgar scores at 1 and $5 \mathrm{~min}$, early onset sepsis, and late onset sepsis defined as culture positive bacterial infection of blood, urine or cerebrospinal fluid that was treated with antibiotics. Respiratory support parameters such as duration of supplemental oxygen therapy and mechanical ventilation provided to infants were also measured. Olsen growth curves (26) were used to stratify infants according to their intrauterine growth status based on BW, sex, and GA.

\section{Cytokine and CRP Analysis}

Up to $0.2 \mathrm{ml}$ of whole blood sample was collected from enrolled infants within $24 \mathrm{~h}$ of birth. Samples were centrifuged at $5,000 \mathrm{rpm}$ for $5 \mathrm{~min}$, and the supernatant serum samples were frozen at $-80^{\circ} \mathrm{C}$ until final analysis. CRP measurements were by enzyme-linked immunosorbent assay (R\&D Systems, Minneapolis, MN). A multiplex Luminex analysis system (Luminex Corporation, Austin, TX) that is commercially available and is able to measure levels of a panel of 39 different cytokines (EMD Millipore, Billerica, MA) was used to measure cytokines (see Table 2). All measurements were reported in $\mathrm{pg} / \mathrm{ml}$.

\section{Outcome Definitions}

The National Institute of Child Health and Human Development physiologic definition was used to identify infants with BPD (27). This method of BPD diagnosis has been well-validated and shown to reduce observer bias. BPD and death by $36 \mathrm{wk}$ PMA were combined together to form the primary study outcome. Thus, "cases" included infants who died before $36 \mathrm{wk}$ or those who survived to $36 \mathrm{wk}$ PMA but developed BPD. Controls were infants who survived to $36 \mathrm{wk}$ PMA without BPD.

\section{Statistical Analysis}

Means with standard deviations (for parametric variables) or medians with ranges (nonparametric variables) were used to summarize continuous variables; proportions were used for categorical variables. Fisher's exact test and chi-square test were used for between-group comparisons of categorical variables. Student's $t$-test, Mann-Whitney test and Kruskal-Wallis test were used for continuous variables. Stepwise logistic regression was used to estimate strength of relationships between categorical outcomes and predictor variables. CRP, cytokines, and clinical variables available by $24 \mathrm{~h}$ of life were used to construct a forward stepwise Classification and Regression Tree (CART) analysis to identify early predictors of 
BPD and/or death using Answertree available in SPSS 2011 (IBM, Chicago, IL). The CART analysis that we used in our study is a robust statistical method that identifies specific levels of various risk factors for a given outcome (say BPD in this instance) by dividing a dataset using recursive partitioning into a classification tree that yields two subgroups-one with a higher proportion of the outcome being tested (cases) and the other with a higher proportion of controls, thereby identifying optimal cutoff points for risk factors in the dataset that allow for such binary division of the given data. All other statistical analyses were carried out with R (R Development Core Team, Vienna, Austria), and Sigmaplot 12.0 (SYSTAT Software, San Jose, CA) was used to generate figures. Two-sided $P$ values less than 0.05 were considered statistically significant. No correction was made for multiple testing in view of the exploratory (hypothesis-generating) nature of these analyses.

\section{STATEMENT OF FINANCIAL SUPPORT}

This study was supported in part by grants from the National Institutes of Health, Bethesda, MD (R03 HD054420, K08 HD 046513, and R01 HL092096) and the American Thoracic Society (ATS), New York, NY/Pulmonary Hypertension Association, Silver Spring, MD (ATS PH-06-006)

Disclosure: There are no conflicts of interest or financial disclosures (other than funding as stated above) for any of the authors.

\section{REFERENCES}

1. Fanaroff AA, Stoll BJ, Wright LL, et al.; NICHD Neonatal Research Network. Trends in neonatal morbidity and mortality for very low birthweight infants. Am J Obstet Gynecol 2007;196:147.e1-8.

2. Bhandari A, Bhandari V. Pitfalls, problems, and progress in bronchopulmonary dysplasia. Pediatrics 2009;123:1562-73.

3. Check J, Gotteiner N, Liu X, et al. Fetal growth restriction and pulmonary hypertension in premature infants with bronchopulmonary dysplasia. J Perinatol 2013;33:553-7.

4. Köksal N, Kayik B, Çetinkaya M, et al. Value of serum and bronchoalveolar fluid lavage pro- and anti-inflammatory cytokine levels for predicting bronchopulmonary dysplasia in premature infants. Eur Cytokine Netw 2012;23:29-35.

5. Munshi UK, Niu JO, Siddiq MM, Parton LA. Elevation of interleukin-8 and interleukin- 6 precedes the influx of neutrophils in tracheal aspirates from preterm infants who develop bronchopulmonary dysplasia. Pediatr Pulmonol 1997;24:331-6.

6. Eklund CM. Proinflammatory cytokines in CRP baseline regulation. Adv Clin Chem 2009;48:111-36.

7. Ambalavanan N, Ross AC, Carlo WA. Retinol-binding protein, transthyretin, and C-reactive protein in extremely low birth weight (ELBW) infants. J Perinatol 2005;25:714-9.

8. Patel RM, Kandefer S, Walsh MC, et al. Causes and timing of death in extremely premature infants from 2000 through 2011. N Engl J Med 2015:372:331-40.

9. Ambalavanan N, Carlo WA, D’Angio CT, et al.; Eunice Kennedy Shriver National Institute of Child Health and Human Development Neonatal Research Network. Cytokines associated with bronchopulmonary dysplasia or death in extremely low birth weight infants. Pediatrics 2009;123:1132-41.
10. Leviton A, Allred EN, Yamamoto H, Fichorova RN; ELGAN Study Investigators. Relationships among the concentrations of 25 inflammation-associated proteins during the first postnatal weeks in the blood of infants born before the $28^{\text {th }}$ week of gestation. Cytokine 2012;57:182-90.

11. Eunice Kennedy Shriver National Institute of Child Health and Human Development Neonatal Research Network. Carlo WA, McDonald SA, Tyson JE, et al. Cytokines and neurodevelopmental outcomes in extremely low birth weight infants. J Pediatr 2011;59:919-925.e913.

12. Yu H, Yuan L, Zou Y, et al. Serum concentrations of cytokines in infants with retinopathy of prematurity. APMIS 2014;122:818-23.

13. Viscardi RM, Muhumuza CK, Rodriguez A, et al. Inflammatory markers in intrauterine and fetal blood and cerebrospinal fluid compartments are associated with adverse pulmonary and neurologic outcomes in preterm infants. Pediatr Res 2004;55:1009-17.

14. Moncunill G, Aponte JJ, Nhabomba AJ, Dobaño C. Performance of multiplex commercial kits to quantify cytokine and chemokine responses in culture supernatants from Plasmodium falciparum stimulations. PLoS One 2013;8:e52587.

15. Yang JY, Cha J, Shim SY, Cho SJ, Park EA. The relationship between eosinophilia and bronchopulmonary dysplasia in premature infants at less than 34 weeks' gestation. Korean J Pediatr 2014;57:171-7.

16. Yamamoto C, Kojima T, Hattori K, et al. Eosinophilia in premature infants: correlation with chronic lung disease. Acta Paediatr 1996;85:1232-5.

17. Yen JM, Lin CH, Yang MM, Hou ST, Lin AH, Lin YJ. Eosinophilia in very low birth weight infants. Pediatr Neonatol 2010;51:116-23.

18. Broström EB, Katz-Salamon M, Lundahl J, Halldén G, Winbladh B. Eosinophil activation in preterm infants with lung disease. Acta Paediatr 2007;96:23-8.

19. Raghavender B, Smith JB. Eosinophil cationic protein in tracheal aspirates of preterm infants with bronchopulmonary dysplasia. J Pediatr 1997;130:944-7.

20. Baier RJ, Loggins J, Kruger TE. Monocyte chemoattractant protein-1 and interleukin-8 are increased in bronchopulmonary dysplasia: relation to isolation of Ureaplasma urealyticum. J Investig Med 2001;49:362-9.

21. Huaux F, Gharaee-Kermani M, Liu T, et al. Role of Eotaxin-1 (CCL11) and CC chemokine receptor 3 (CCR3) in bleomycin-induced lung injury and fibrosis. Am J Pathol 2005;167:1485-96.

22. Arnon S, Grigg J, Silverman M. Pulmonary inflammatory cells in ventilated preterm infants: effect of surfactant treatment. Arch Dis Child 1993;69(1 Spec No):44-8.

23. Jamaluddin MS, Wang X, Wang H, Rafael C, Yao Q, Chen C. Eotaxin increases monolayer permeability of human coronary artery endothelial cells. Arterioscler Thromb Vasc Biol 2009;29:2146-52.

24. Haley KJ, Sunday ME, Porrata Y, et al. Ontogeny of the eotaxins in human lung. Am J Physiol Lung Cell Mol Physiol 2008;294:L214-24.

25. Batra J, Rajpoot R, Ahluwalia J, et al. A hexanucleotide repeat upstream of eotaxin gene promoter is associated with asthma, serum total IgE and plasma eotaxin levels. J Med Genet 2007;44:397-403.

26. Olsen IE, Groveman SA, Lawson ML, Clark RH, Zemel BS. New intrauterine growth curves based on United States data. Pediatrics 2010;125: e214-24.

27. Walsh MC, Wilson-Costello D, Zadell A, Newman N, Fanaroff A. Safety, reliability, and validity of a physiologic definition of bronchopulmonary dysplasia. J Perinatol 2003;23:451-6. 